\title{
Computer simulation of turbulent reactive gas dynamics
}

\author{
BJØRN H. HJERTAGER $\dagger$
}

Keywords: Gas explosion, Fluid dynamics, Combustion

A simulation procedure capable of handling transient compressible flows involving combustion is presented. The method uses the velocity components and pressure as primary flow variables. The differential equations governing the flow are discretized by integration over control volumes. The integration is performed by application of up-wind differencing in a staggered grid system. The solution procedure is an extension of the SIMPLE-algorithm accounting for compressibility effects.

Turbulence is treated by solving the equations of kinetic energy of turbulence and of dissipation rate of kinetic energy of turbulence. The combustion model incorporates solution of balance equations for the mixture fraction and the mass fraction of fuel. The rate of combustion in the latter equation is modeled according to the 'eddy-dissipation model' of Magnussen and Hjertager (1976). A simple criteria is introduced to enable chemical kinetics to play a role in limiting the rate of combustion.

A validation calculation of the classical shock-tube problem is performed and good agreement between analytical solution and computed predictions is found.

Flame acceleration by repeated obstacles in methane-air and propane-air are also simulated, and comparisons with experimental data are encouraging.

\section{Introduction}

\subsection{Statement of problem}

Simulation of combustion processes in turbulent and compressible flows is a complex problem of both fundamental and practical importance. The non-linearities and range of time scales in the problem makes it a demanding task from both computational and conceptual points of view. Adequate modeling of the coupling of fluid dynamics and reaction rate is the key to successful prediction of e.g. turbulent flame propagation, pollutant formation and overall efficiency of various combustion processes.

The shortage of fossil fuels has focused attention on more efficient use of these resources. To improve performance of, and minimize pollution from combustion devices, there is a need for good predictive tools, with the objective to identify the optimum design or mode of operation. Furthermore, the increased production and thus transport and handling of large quantities of flammable gases has increased the need for adequate means of predicting flame and pressure development in possible accidental explosions, and thus enable adoption of realistic safety measures.

Received 10 July 1984.

$\dagger$ Chr. Michelsen Institute, Department of Science and Technology, N-5036, Fantoft, Bergen, Norway. 


\subsection{Previous work}

Several prediction methods capable of handling transient compressible flows with chemical reactions have been published. All of these methods use explicit formulation of the momentum and scalar equations. Explicit formulation indicates that calculation of advanced time values of the dependent variables are obtained without solution of simultaneous algebraic equations. The method of Butler and O'Rourke (1976) uses the so-called ICE (Implicit Continuous-fluid-Eulerian) technique to eliminate the Courant sound speed criterion. This method, together with the ALE (Arbitrary Lagrangian Eulerian) technique, is used by Boni et al. (1976) to study combustion in the stratified charge engine. Oran et al. (1978) have used a fully explicit scheme to study the details of detonation waves. A special feature of their problem is to resolve and maintain steep gradients in the flow. This was accomplished by use of the non-linear FCT (Flux Corrected Transport) technique. All methods mentioned above have time step limitation due to their explicit formulation. This may be a disadvantage if slow variations are studied or if a steady-state solution is sought. An alternative formulation is to use implicit methods. These methods are characterized by finding the time advanced values of the dependent variables by solving a set of simultaneous algebraic equations. In principle these do not have any time step limitations and may therefore also be used for steady state incompressible as well as compressible turbulent flows.

\subsection{Purpose of paper}

The present paper reviews a computation method based on the Patankar and Spalding (1972) method in the form given by Hjertager and Magnussen (1976). This method was initially developed for incompressible reacting flows and has subsequently been extended by Hjertager (1982a) to take account of compressible flows. The proposed modeling technique will be tested against the analytical shock tube solution. Some applications to explosion propagation in propane-air and methaneair mixtures will be given. Predicted scaling behaviour of the explosion problem will also be shown.

\subsection{Contents of paper}

Section 2 of this paper formulates the basic equations to be solved. Sections 3, 4 and 5 outline the various models adopted, namely turbulence, combustion and thermodynamics, respectively. Section 6 gives the details of the numerical calculation method. Section 7 gives several sample calculations of the numerical method. Section 8 deals with the conclusions.

\section{Governing equations}

The problem of turbulent transient flows can be handled by solving for the time evolution of time mean values of the dependent quantities, $\Phi$, in the domain of interest. The time mean of a variable varying with time, $t$, may be expressed as:

$$
\Phi(t)=\frac{1}{T} \int_{t}^{t+T} \hat{\Phi}(t+\tau) d \tau
$$


where $\Phi(t)$ is the time mean value of the instantaneous value $\hat{\Phi}(t)$ averaged over the time interval T.T must satisfy two competing demands. First, it must be small enough not to smear out the sought time dependence of the system under consideration. Secondly, it must be large enough to be able to produce sufficient information to enable relevant time mean values in the interval. This means time mean values of both the relevant quantities and their second order correlations must be obtainable in the time interval $T$. This is often possible since conversely, turbulence has higher frequencies than the large scale motion which generates turbulence. The equation of motion and energy may be expressed in tensor notation as:

$$
\begin{aligned}
\frac{\partial \rho}{\partial t}+\frac{\partial}{\partial x_{i}}\left(\rho U_{i}\right) & =0 \\
\frac{\partial}{\partial t}\left(\rho U_{i}\right)+\frac{\partial}{\partial x_{j}}\left(\rho U_{j} U_{i}\right) & =-\frac{\partial p}{\partial x_{i}}+\frac{\partial}{\partial x_{j}}\left(\sigma_{i j}\right)+\rho g_{i} \\
\frac{\partial}{\partial t}(\rho h)+\frac{\partial}{\partial x_{j}}\left(\rho U_{j} h\right) & =-\frac{\partial}{\partial x_{j}}\left(J_{h, j}\right)+\frac{D p}{D t}+S_{h}
\end{aligned}
$$

Here $U_{i}$ is the velocity component in the $x_{i}$ coordinate direction; $g_{i}$ is the gravitation in $x_{i}$ direction; $p$ is the pressure; $\rho$ is the density; $h$ is the enthalpy and $\sigma_{i j}$ and $J_{h, j}$ are the fluxes of momentum and energy. $S_{h}$ is an additional source term which may include such sources as radiation influences and viscous dissipation. The conservation equation for a specie is given as

$$
\frac{\partial}{\partial t}\left(\rho m_{i}\right)+\frac{\partial}{\partial x_{j}}\left(\rho U_{j} m_{i}\right)=-\frac{\partial}{\partial x_{j}}\left(J_{i, j}\right)+R_{i}
$$

where $R_{i}$ is the net reaction rate of the specie $m_{i}$.

\section{Turbulence model}

\subsection{Closure assumptions}

To solve the governing eqns. (2), (3), (4) and (5) given above, the fluxes, $J_{\Phi, j}$, and the net rate of reaction $R_{i}$, have to be modeled together with specification of relevant boundary and initial conditions. Both the fluxes and the reaction rate are time mean averaged values of fluctuating quantities. The fluxes can, for a general scalar variable $\Phi$, and a velocity component $U_{j}$, be expressed as:

and

$$
J_{\Phi, j}=-\rho \overline{u_{j} \varphi}
$$

$$
\sigma_{i j}=\rho \overline{u_{i} u_{j}}
$$

where $u_{i}$ and $\varphi$ are the instantaneous fluctuations around the time mean values $U_{j}$ and $\Phi$, respectively. The overbar indicates time mean value over the time interval $T$ as defined in expression (1). When specifying the correlations given in (6) and (7) it is usual to relate these to the product of time mean gradients of the relevant variables and an effective turbulent transport coefficient. For a general scalar variable $\Phi$ and a velocity component $U_{j}$ the relations are:

$$
J_{\Phi, j}=-\frac{\mu_{\mathrm{eff}}}{\sigma_{\Phi}} \frac{\partial \Phi}{\partial x_{j}}
$$


and

$$
\sigma_{i j}=\mu_{\mathrm{eff}}\left(\frac{\partial U_{i}}{\partial x_{j}}+\frac{\partial U_{j}}{\partial x_{i}}\right)-\frac{2}{3} \delta_{i j}\left(\rho k+\mu_{\mathrm{eff}} \frac{\partial U_{k}}{\partial x_{k}}\right)
$$

respectively.

Here $\delta_{i j}=1$ if $i=j$ and $\delta_{i j}=0$ if $i \neq j$. An effective turbulence viscosity $\mu_{\mathrm{eff}}$ and the kinetic energy of turbulence have been introduced in the above expressions. together with an effective Prandtl/Schmidt number $\sigma_{\Phi}$. The kinetic energy of turbulence, $k$, is related to the fluctuating turbulence velocity components in the three coordinate directions as:

$$
k=\frac{1}{2}\left(\overline{u_{1}^{2}}+\overline{u_{2}^{2}}+\overline{u_{3}^{2}}\right)
$$

The effective turbulence viscosity is given by the two turbulence parameters, the turbulence velocity $u_{t}$ and a length scale, $l$, as:

$$
\mu_{\text {eff }}=\mu_{1}+\rho u_{t} l
$$

$\mu_{1}$ is the molecular viscosity.

\subsection{Two-parameter turbulence model}

The determination of $u_{t}$ and $l$ are at present best done by application of the so-called $k-\varepsilon$ model of turbulence given by Launder and Spalding (1974). The turbulence velocity is related to the kinetic energy of turbulence, $k$, as:

$$
u_{t}=\left(\frac{2}{3} k\right)^{1 / 2}
$$

and the length scale, $l$, is related to the kinetic energy of turbulence, $k$, and its rate of dissipation, $\varepsilon$, as:

$$
l \sim \frac{k^{3 / 2}}{\varepsilon}
$$

Inserting (12) and (13) into expression (11) give as result:

$$
\mu_{\mathrm{eff}}=\mu_{1}+C_{D} \rho \frac{k^{2}}{\varepsilon}
$$

$C_{D}$ is a constant taken to be 0.09 .

The conservation equations that determine the distribution of $k$ and $\varepsilon$ read:

$$
\begin{aligned}
& \frac{\partial \rho k}{\partial t}+\frac{\partial}{\partial x_{j}}\left(\rho U_{j} k\right)=\frac{\partial}{\partial x_{j}}\left(\frac{\mu_{\mathrm{eff}}}{\sigma_{k}} \frac{\partial k}{\partial x_{j}}\right)+G-\rho \varepsilon \\
& \frac{\partial \rho \varepsilon}{\partial t}+\frac{\partial}{\partial x_{j}}\left(\rho U_{j} \varepsilon\right)=\frac{\partial}{\partial x_{j}}\left(\frac{\mu_{\mathrm{eff}}}{\sigma_{\varepsilon}} \frac{\partial \varepsilon}{\partial x_{j}}\right)+C_{1} \frac{\varepsilon}{k} G-C_{2} \rho \frac{\varepsilon^{2}}{k}
\end{aligned}
$$

The two new constants appearing above $C_{1}$ and $C_{2}$ are given the values 1.44 and 1.79 , respectively. The Schmidt numbers $\sigma_{k}$ and $\sigma_{\varepsilon}$ are given the values 1.0 and 1.3 , respectively, whereas the other Schmidt/Prandtl numbers are put equal to 0.7. All the constants given above are taken from the paper by Launder and Spalding (1974). The model constants have been verified for a range of flows, both isothermal 
and combusting situations (Launder and Spalding, 1974). The generation rate of turbulence is given by:

$$
G=\sigma_{i j} \frac{\partial U_{j}}{\partial x_{i}}
$$

These terms are related to turbulence production due to shear and expansion/ compression.

\section{Combustion modeling}

The combustion process is treated as a single step irreversible reaction with finite reaction rate between fuel and oxydant. Hence, the reaction scheme may be written as:

$$
1 \mathrm{~kg} \text { fuel }+s \mathrm{~kg} \text { oxygen } \rightarrow(1+s) \mathrm{kg} \text { products }
$$

Here $s$ is the stoichiometric oxygen requirement to burn $1 \mathrm{~kg}$ of fuel. This simple reaction scheme results in mixture composition being determined by solving for only two variables, namely mass fraction of fuel, $m_{f u}$, and the mixture fraction, $f$ :

$$
\begin{aligned}
\frac{\partial}{\partial t}\left(\rho m_{f u}\right)+\frac{\partial}{\partial x_{j}}\left(\rho U_{j} m_{f u}\right) & =-\frac{\partial}{\partial x_{j}}\left(J_{f u, j}\right)+R_{f u} \\
\frac{\partial}{\partial t}(\rho f)+\frac{\partial}{\partial x_{j}}\left(\rho U_{j} f\right) & =-\frac{\partial}{\partial x_{j}}\left(J_{f, j}\right)
\end{aligned}
$$

Here $R_{f u}$ is the time mean rate of combustion of fuel, whereas $J_{f u, j}$ and $J_{f, j}$ are the diffusive fluxes in the $x_{j}$-direction. The basis for this to be valid is that the effective turbulent Schmidt numbers are equal for all species, an approximation which is often found to be valid in turbulent flows.

The mixture fraction is defined as:

$$
f=\frac{\beta-\beta_{\infty}}{\beta_{\mathrm{o}}-\beta_{\infty}}
$$

where $\beta$ is a conserved combined variable of for example mass fraction of fuel, $m_{f u}$, and mass fraction of oxygen, $m_{\mathrm{O}_{2}}$, expressed as:

$$
\beta=m_{f u}-m_{\mathrm{O}_{2}} / s
$$

$\beta_{\mathrm{O}}$ is the value of $\beta$ at a fuel rich reference point, for example a fuel inlet point in the domain, and $\beta_{\infty}$ is the value of $\beta$ at an oxydant rich reference point, for example the ambient air condition. For a homogeneous premixed system the mixture fraction will be constant in the domain of interest and consequently only the $m_{f u}$-equation needs to be solved.

For the simple reaction scheme given in (18) above an instantaneous rate of reaction can be written as:

$$
\hat{R}_{f u}=-\rho^{2} \hat{k}_{f u} \hat{m}_{f u} \hat{m}_{\mathrm{O}_{2}}
$$

Here $\hat{k}_{f u}$ is the instantaneous value of the Arrhenius temperature dependence of the rate constant. If this rate constant and the mass fractions are written as the sum of a time mean and a fluctuating quantity $\left(\hat{m}_{i}=m_{i}+m_{i}^{\prime}\right)$, the reaction rate (ignoring 
density fluctuations) would be written as:

$$
\hat{R}_{f u}=-\rho^{2}\left(k_{f u}+k_{f u}^{\prime}\right)\left(m_{f u}+m_{f u}^{\prime}\right)\left(m_{\mathrm{O}_{2}}+m_{\mathrm{O}_{2}}^{\prime}\right)
$$

Multiplying out this expression and taking the time mean value of the result gives:

$$
R_{f u}=-\rho^{2}\left(k_{f u} m_{f u} m_{\mathrm{O}_{2}}+k_{f u} \overline{m_{f u}^{\prime} m_{\mathrm{O}_{2}}^{\prime}}+m_{f u} \overline{k_{f u}^{\prime} m_{\mathrm{O}_{2}}^{\prime}}+m_{\mathrm{O}_{2}} \overline{k_{f u}^{\prime} m_{f u}^{\prime}}+\overline{k_{f u}^{\prime} m_{f u}^{\prime} m_{\mathrm{O}_{2}}^{\prime}}\right)
$$

The above relation shows that even the simplest reaction scheme gives rise to a rather complicated expression for the time mean reaction rate. This indicates that rigorous mathematical methods of attack of the reaction rate based on chemical kinetics in turbulent situations have immense obstacles prior to providing solutions. It is therefore necessary to seek, if possible, alternative and simpler methods.

Experiments (Spalding, 1971) have shown that the rate of combustion in flames is mainly dependent on hydrodynamic parameters. This implies that combustion rate is limited by the rate of molecular mixing between the reactants. This mixing is directly linked to the rate at which turbulent eddies are dissipated. For a scalar variable this dissipation is formally expressed as:

$$
\varepsilon_{\Phi}=D_{\Phi} \frac{\partial \Phi}{\partial x_{k}} \cdot \frac{\partial \Phi}{\partial x_{k}}
$$

$D_{\Phi}$ is the molecular diffusion coefficient. The hydrodynamic rate of dissipation of turbulent kinetic energy is denoted by $\varepsilon$. It is therefore assumed that combustion rate is proportional to the rate of dissipation of kinetic energy of turbulence

$$
R_{f u}=-\rho \varepsilon \frac{g_{\Phi}^{1 / 2}}{k}
$$

$g_{\Phi}$ is the variance of the fluctuations of the limiting specie in question. Magnussen and Hjertager (1976) argue that the fuel, oxydant and reaction products appear as intermittent fluctuating quantities. Consequently, the fluctuating species may be related to time mean values of fuel, oxydant or reaction products. Therefore:

$$
R_{f u}=-A \rho \frac{\varepsilon}{k} m_{\text {lim }}
$$

where $m_{\text {lim }}$ is the smallest of the three mass fractions, namely fuel, $m_{f u}$, oxygen $m_{\mathrm{O}_{2}} / s$ or mass fraction of fuel already burnt, $m_{f u, b}$. $A$ is a constant. In order for expression (28) to be valid, the chemical kinetics of the system under consideration must be fast. In many cases this is not the case, especially in the fast transient combustion which, for example, occurs in high speed gas explosions.

A simple modification of the above expression has therefore been proposed by Hjertager (1982b). Based on the chemical kinetics of the system, a chemical time can be defined, $\tau_{c h}$. Also, the lifetime of the turbulent eddies can be defined, $\tau_{e}$. Ignition or extinction is assumed to occur when these two times are in a given ratio, namely:

$$
\tau_{c h} / \tau_{e}=D_{i e}
$$

The following modification to the rate expression in (28) is therefore used:

$$
\left.\begin{array}{lll}
\text { if } \quad \tau_{c h} / \tau_{e} \geq D_{i e} & \text { then } \quad R_{f u}=0 \\
\text { if } \quad \tau_{c h} / \tau_{e}<D_{i e} & \text { then } \quad R_{f u}=-A \rho \frac{\varepsilon}{k} m_{\lim }
\end{array}\right\}
$$


The above criteria is closely related to the models proposed by Radhakrishnan et al. (1981) and Magnussen (1981) for extinction phenomena. The eddy lifetime or mixing time is defined as:

$$
\tau_{e}=\frac{l}{u_{t}} \sim \frac{k}{\varepsilon}
$$

The chemical time is taken equal to the chemical induction time which is often expressed as:

$$
\tau_{c h}=A_{c h} \exp (E / R T)\left(\rho m_{f u}\right)^{a}\left(\rho m_{\mathrm{O}_{2}}\right)^{b}
$$

Radhakrishnan et al. (1981) proposed that the chemical time should be taken equal to the time for a laminar flame to propagate across a length equal to the Taylor micro scale, $\lambda$. Their application, however, was a correlation of blow-off velocity data in disc-stabilized premixed flames burning in constant pressure environment. Our application, however, is to systems with compression and expansion pressure waves where the chemical induction times are the more appropriate choice.

\section{Thermodynamics modeling}

For a single-step reaction of the form given in (18) above, a total of five mass fractions may be identified, namely; fuel, nitrogen, oxygen, carbon dioxide and water vapour.

The density of the gas mixture can be calculated from the ideal gas law as:

$$
\rho=\frac{M_{a v} p}{R_{u} T}
$$

where the average molecular weight is calculated from:

$$
\frac{1}{M_{a v}}=\sum_{i=1}^{5} \frac{m_{i}}{M_{i}}
$$

The enthalpy given in eqn. 4 above is defined as:

$$
h=c_{p m, \text { mix }} T+m_{f u} H_{f u}
$$

Here, the mixture specific heat is calculated from each of the gases present in the mixture as:

$$
c_{p m, \text { mix }}=\sum_{i=1}^{5} m_{i} c_{p m, i}
$$

where

$$
c_{p m, i}=\frac{1}{T-T_{\mathrm{ref}}} \int_{T_{\mathrm{ref}}}^{T} c_{p, i} d T
$$

The specific heats are assumed to vary linearly with temperature:

$$
c_{p m, i}=a_{i}+b_{i} T
$$

Data for the $a_{i} \mathrm{~s}$ and $b_{i} \mathrm{~s}$ are taken from Khalil et al. (1975). 


\section{Numerical procedure}

\subsection{Finite-domain equations}

It is noted that all conservation equations mentioned above can be written in the following general form:

$$
\frac{\partial}{\partial t}(\rho \Phi)+\frac{\partial}{\partial x_{j}}\left(\rho U_{j} \Phi\right)=\frac{\partial}{\partial x_{j}}\left(\Gamma_{\Phi} \frac{\partial \Phi}{\partial x_{j}}\right)+S_{\Phi} ; \quad \Gamma_{\Phi}=\frac{\mu_{\text {eff }}}{\sigma_{\phi}}
$$

$$
\text { I II III IV }
$$

This means, equations with four distinct terms, namely: I transient, II convection, III diffusion and IV source terms. A summary of all the equations needed for a typical reactive gas dynamic calculation are given in Table $\mathbf{I}$.

The calculation domain is divided into a discrete number of control volumes surrounding a grid point. The staggered grid has been employed and is shown in Fig. 1. The figure shows a central point $\mathrm{P}$ surrounded by its neighbouring points $\mathrm{E}$, $\mathrm{W}, \mathrm{N}, \mathrm{S}$ in the $x y$-plane and $\mathrm{H}, \mathrm{L}$ normal to this plane (not shown). All dependent variables except velocity components are stored at the grid line intersections (nodes), whereas velocity components are stored midway between these nodes. Locations between the main grid nodes are denoted by lower case letters. For example, the location between node $\mathrm{P}$ and $\mathrm{S}$ will be denoted by $s$.

\begin{tabular}{|c|c|c|}
\hline Quantity & Stored at & Control Volume \\
\hline$p, h, m_{f u}, f, k, \varepsilon$ & 0 & \\
\hline U-velocity & $D$ & \\
\hline V-velocity & $\Delta$ & \\
\hline
\end{tabular}

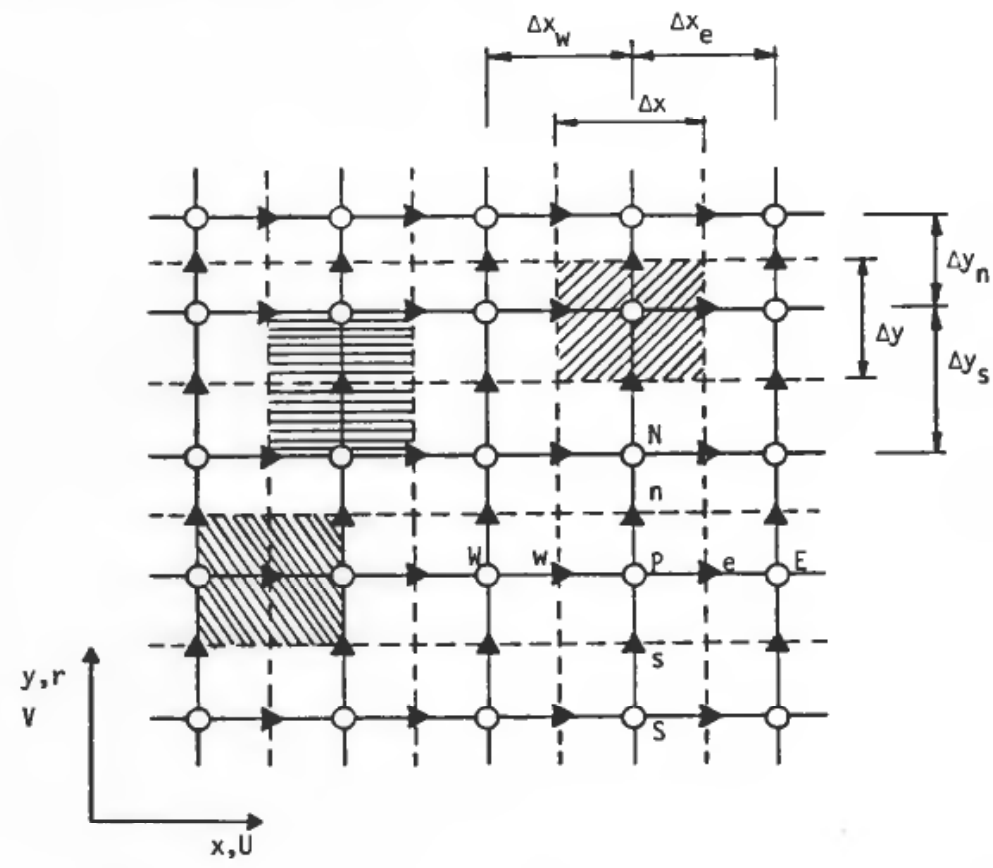

Figure 1. Grid layout and notation. 


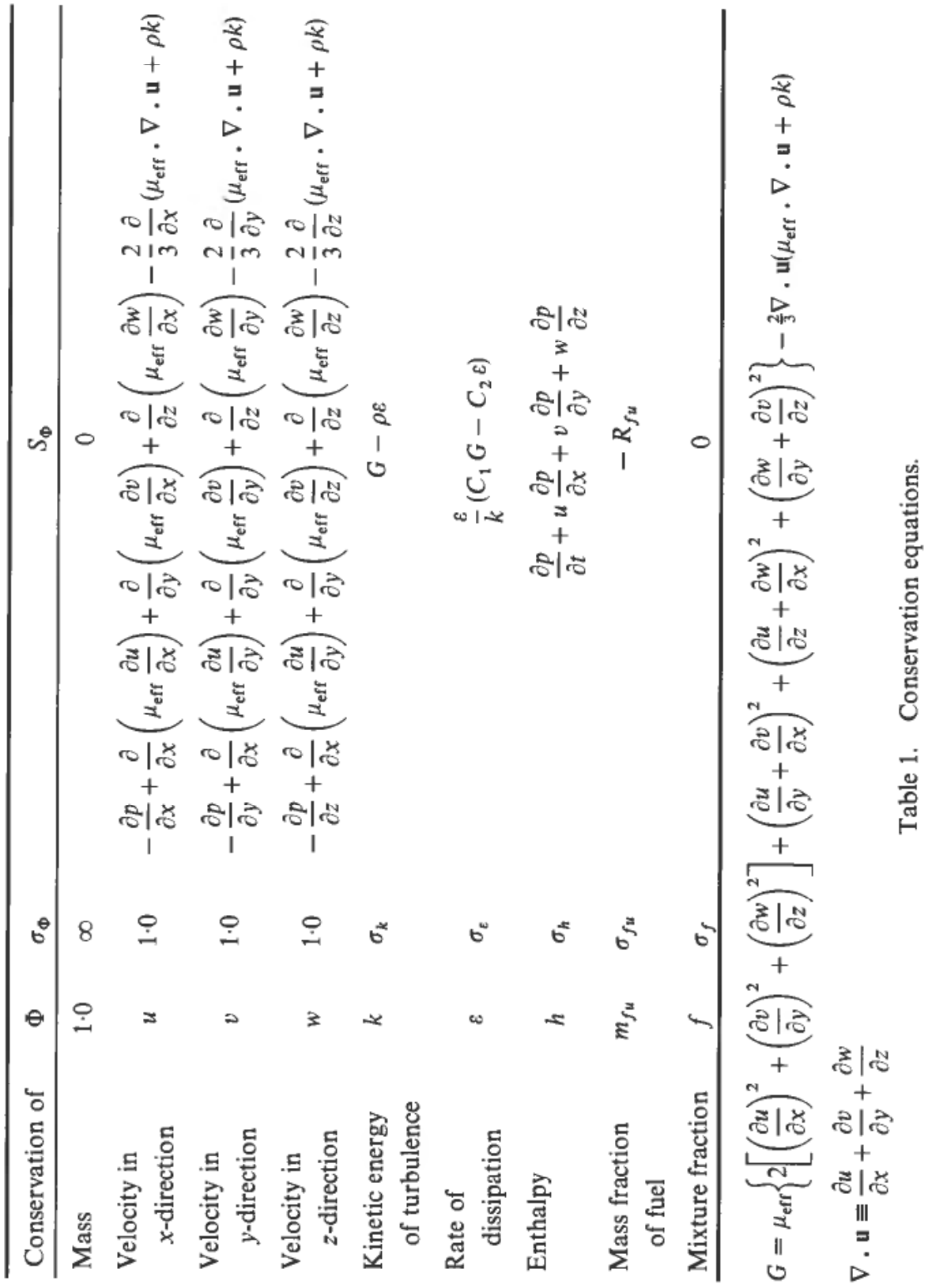




\subsubsection{Finite-domain approximations}

In order to solve the governing equations, these are integrated over a control volume. If we, for instance, take the equation for a scalar quantity, the proper control volume is shown in Fig. 1. The net diffusive and convective flux in the $y$-direction is expressed by:

$$
A_{\mathrm{N}}^{\Phi}\left(\Phi_{\mathrm{P}}-\Phi_{\mathrm{N}}\right)+A_{\mathrm{S}}^{\Phi}\left(\Phi_{\mathrm{P}}-\Phi_{\mathrm{S}}\right)
$$

The coefficients are:

$$
\begin{aligned}
& A_{\mathrm{N}}^{\Phi}=\left(\Gamma_{\phi, n} / \Delta y_{n}+\left\langle-\rho_{n} V_{\mathrm{N}}\right\rangle\right) \Delta x \Delta z \\
& A_{\mathrm{S}}^{\Phi}=\left(\Gamma_{\phi, s} / \Delta y_{s}+\left\langle\rho_{s} V_{\mathrm{P}}\right\rangle\right) \Delta x \Delta z
\end{aligned}
$$

Here, the expression in \langle\rangle is the upwind differencing for the convection terms expressed by:

$$
\langle e\rangle=0 \cdot 5(e+|e|)
$$

This means that the value of $\Phi$ convected into or out of the control volume always has its upstream value.

The time derivative is expressed by:

$$
b^{\phi}\left(\phi_{\mathbf{P}}-\phi_{\mathbf{P}}^{0}\right)
$$

where

$$
b^{\phi}=\rho_{\mathrm{p}}^{0} \Delta x \Delta y \Delta z / \Delta t
$$

and $\phi_{\mathrm{p}}^{0}$ is the value of $\Phi$ at point $\mathrm{P}$ at the previous time level.

Usually it is common to linearize the source term so that

$$
S^{\phi}=S_{0}^{\phi}+S_{\mathbf{P}}^{\phi} \cdot \Phi_{\mathbf{P}}
$$

Here $S^{\phi}, S_{0}^{\phi}$ and $S_{\mathrm{P}}^{\phi}$ are the volume integral of the source term given in (38).

\subsubsection{Domain equations}

Similar expressions as (39) can be derived for the net fluxes in the other two directions. This eventually gives the finite-domain equivalent to eqn. (38):

$$
A_{\mathrm{P}}^{\phi} \Phi_{\mathrm{P}}=A_{\mathrm{N}}^{\phi} \Phi_{\mathrm{N}}+A_{\mathrm{S}}^{\phi} \Phi_{\mathrm{S}}+A_{\mathrm{H}}^{\phi} \Phi_{\mathrm{H}}+A_{\mathrm{L}}^{\phi} \phi_{\mathrm{L}}+A_{\mathrm{E}}^{\phi} \phi_{\mathrm{E}}+A_{\mathrm{W}}^{\phi} \phi_{\mathrm{W}}+S_{0}^{\phi}+b^{\phi} \phi_{\mathrm{P}}^{0}
$$

Here

$$
A_{\mathrm{P}}^{\phi}=A_{\mathrm{N}}^{\phi}+A_{\mathrm{S}}^{\phi}+A_{\mathrm{H}}^{\phi}+A_{\mathrm{L}}^{\phi}+A_{\mathrm{E}}^{\phi}+A_{\mathrm{W}}^{\phi}+b^{\phi}-S_{\mathrm{P}}^{\phi}
$$

In order to secure stability of the numerical scheme, $S_{\mathrm{P}}^{\phi}$ should always be chosen negative.

The momentum eqns. (3) can be integrated in a similar way to the scalar equations. The only difference is that the control volume is different. For $U$ - and $V$ velocities the proper volumes are shown in Fig. 1. The finite domain equivalent for the momentum equations can thus be written as:

$$
\begin{aligned}
& A_{\mathrm{P}}^{U} U_{\mathrm{P}}=\sum_{i} A_{i}^{U} U_{i}+b^{U} U_{\mathrm{P}}^{0}+\Delta y \Delta z\left(p_{\mathrm{W}}-p_{\mathrm{P}}\right)+S_{0}^{U} \\
& A_{\mathrm{P}}^{V} V_{\mathrm{P}}=\sum_{i} A_{i}^{V} V_{i}+b^{V} V_{\mathrm{P}}^{0}+\Delta x \Delta z\left(p_{\mathrm{s}}-p_{\mathrm{P}}\right)+S_{0}^{V} \\
& A_{\mathrm{P}}^{W} W_{\mathrm{P}}=\sum_{i} A_{i}^{W} W_{i}+b^{W} W_{\mathrm{P}}^{0}+\Delta x \Delta y\left(p_{\mathrm{L}}-p_{\mathrm{P}}\right)+S_{0}^{W}
\end{aligned}
$$


The summation is performed around the neighbouring points $\mathrm{N}, \mathrm{S}, \mathrm{H}, \mathrm{L}, \mathrm{E}, \mathrm{W}$ as was shown in equation (45). The constant $A_{i}^{i}$ is also expressed in the same manner as in equation (46).

Finally, the finite-domain approximation for the continuity equation is obtained by integrating it over the control volume in Fig. 1. Thus:

$$
\begin{aligned}
\left(\rho_{\mathrm{P}}-\rho_{\mathrm{P}}^{0}\right) / \Delta t \cdot(\Delta x \Delta y \Delta z)+ & \left((\rho U)_{e}-(\rho U)_{w}\right) \Delta y \Delta z+\left((\rho V)_{n}\right. \\
& \left.-(\rho V)_{s}\right) \Delta x \Delta z+\left((\rho W)_{h}-(\rho W)_{l}\right) \Delta x \Delta y=0
\end{aligned}
$$

\subsection{Solution procedure}

To solve the eqns. (45) to (50), the SIMPLE method (Semi-Implicit Method for Pressure Linked Equations) of Patankar and Spalding (1972) has been adopted. The procedure involves a series of "guess and correct" operations for the coupling of pressure with velocities. A description of the method will now be given which also includes the handling of compressibility presented by Hjertager (1982a).

To start in a forward time step, we calculate all coefficients in the finite-domain eqns. (45) to (50) and these are held constant during the step. Next, a pressure field at the new time level has to be guessed. This can be given, or the result from the previous time level can be used. This field can be denoted by $p^{*}$. With this field we can solve the momentum eqns. (47), (48) and (49) to give intermediate velocity fields at the new time level and, from the ideal gas law (33), we can calculate the intermediate density field. These fields can be denoted by $U^{*}, V^{*}, W^{*}$ and $\rho^{*}$ and will in general not satisfy the continuity eqn. (50). To get the velocity and density fields in accordance with continuity, the following corrections are performed:

$$
\begin{aligned}
U_{\mathrm{P}} & =U_{\mathrm{P}}^{*}+D_{\mathrm{P}}^{U}\left(p_{\mathrm{W}}^{\prime}-p_{\mathrm{P}}^{\prime}\right) \\
V_{\mathrm{P}} & =V_{\mathrm{P}}^{*}+D_{\mathrm{P}}^{U}\left(p_{\mathrm{S}}^{\prime}-p_{\mathrm{P}}^{\prime}\right) \\
W_{\mathbf{P}} & =W_{\mathrm{P}}^{*}+D_{\mathrm{P}}^{W}\left(p_{\mathbf{L}}^{\prime}-p_{\mathrm{P}}^{\prime}\right) \\
\rho_{\mathrm{P}} & =\rho_{\mathrm{P}}^{*}+\left.\frac{\partial \rho}{\partial p}\right|_{\mathrm{P}} p_{\mathrm{P}}^{\prime} \\
p_{\mathrm{P}} & =p_{\mathrm{P}}^{*}+p_{\mathrm{P}}^{\prime}
\end{aligned}
$$

Here a new variable $p^{\prime}$, the pressure correction, is introduced. The constants $D$ come from the linearized momentum equations and are defined as:

$$
D_{\mathrm{P}}^{U}=\Delta y \Delta z / A_{\mathrm{P}}^{U} ; \quad D_{\mathrm{P}}^{V}=\Delta x \Delta z / A_{\mathrm{P}}^{V} \quad \text { and } \quad D_{\mathrm{P}}^{W}=\Delta x \Delta y / A_{\mathrm{P}}^{W}
$$

Density variation with pressure is taken to be isentropic. This leads to

$$
\left.\frac{\partial \rho}{\partial p}\right|_{\mathbf{P}}=\frac{1}{c_{s, \mathrm{P}}^{2}}
$$

where $c_{s, \mathrm{P}}$ is the local sound speed at point $\mathrm{P}$.

To determine the pressure correction $p^{\prime}$ the expressions (51) to (54) are substituted into the continuity, equation (50), thus giving the following finite-domain equation for $p^{\prime}$ :

$$
A_{\mathrm{P}}^{p} p_{\mathrm{P}}^{\prime}=\sum_{k} A_{k}^{p} p_{k}^{\prime}+S^{p}
$$


The summation is again performed around the 6 surrounding points. Further, the coefficients are expressed as follows:

$$
A_{\mathrm{P}}^{p}=\sum_{k} A_{k}^{p} ; \quad A_{\mathrm{W}}^{p}=\Delta y \Delta z\left[\rho_{w} D_{\mathrm{P}}^{U}+\frac{1}{c_{s, w}^{2}} \cdot\left\langle U_{p}\right\rangle\right] \text { etc. }
$$

The source term is:

$$
\begin{aligned}
S^{p}=\left(\left(\rho U^{*}\right)_{w}-\left(\rho U^{*}\right)_{e}\right) \Delta y \Delta z & +\left(\left(\rho V^{*}\right)_{s}-\left(\rho V^{*}\right)_{n}\right) \Delta x \Delta z \\
& +\left(\left(\rho W^{*}\right)_{l}-\left(\rho W^{*}\right)_{h}\right) \Delta x \Delta y+\left(\rho_{\mathrm{P}}^{0}-\rho_{\mathrm{P}}^{*}\right) / \Delta t \cdot \Delta x \Delta y \Delta z
\end{aligned}
$$

We can see that the source term is the local unbalance in the continuity equation of the intermediate velocity fields.

When equation (56) for the pressure corrections has been solved, the corrections (51) to (55) can be performed and, if the resulting mass unbalance is sufficiently small, then the values of the variables at the new time level have been found. If the mass unbalance is too large, repeated solution of equation (56) and corrections (51) to (55) must be performed.

\subsubsection{Solution of the algebraic equations}

In the preceeding section we have referred to solving the equations for momentum and pressure correction. The actual method of solving is performed by the well known tridiagonal matrix algorithm. This algorithm is used successively in each of the three coordinate directions. At each sweep, the variables along a line are assumed to be unknown whereas the variables in the surrounding lines are assumed to be known. This practice results in the variable at a point only being connected with variables at two neighbouring points along the line in question. A triple sweep will thus consist of the three following stages.

Stage I:

$A_{\mathrm{P}}^{\phi} \Phi_{\mathrm{P}}^{\mathrm{I}}=A_{\mathrm{N}}^{\phi} \Phi_{\mathrm{N}}^{\mathrm{I}}+A_{\mathrm{S}}^{\phi} \Phi_{\mathrm{S}}^{\mathrm{I}}+F_{\mathrm{I}}\left(\Phi^{0}, S_{\mathrm{o}}^{\phi}\right)$

Stage II:

$$
\left.\begin{array}{l}
A_{\mathrm{P}}^{\phi} \Phi_{\mathrm{P}}^{\mathrm{II}}=A_{\mathrm{H}}^{\phi} \Phi_{\mathrm{H}}^{\mathrm{II}}+A_{\mathrm{L}}^{\phi} \Phi_{\mathrm{L}}^{\mathrm{II}}+F_{\mathrm{II}}\left(\Phi^{0}, \Phi^{\mathrm{l}}, S_{\mathrm{O}}^{\phi}\right) \\
\text { Stage III: } \\
A_{\mathrm{P}}^{\phi} \Phi_{\mathrm{P}}^{*}=A_{\mathrm{E}}^{\phi} \Phi_{\mathrm{E}}^{*}+A_{\mathrm{W}}^{\phi} \Phi_{\mathrm{W}}^{*}+F_{\mathrm{III}}\left(\Phi^{0}, \Phi^{\mathrm{I}}, \Phi^{\mathrm{II}}, S_{\mathrm{o}}^{\phi}\right)
\end{array}\right\}
$$

One such triple sweep is performed for velocity components and $\Phi$ s while three are done on the pressure correction $\left(p^{\prime}\right)$. In many cases the so-called block correction may also be included in the solution of the equations. Details of this technique may be found in the paper by Patankar (1981).

\subsubsection{Boundary conditions}

Because we are working with the primitive variables $U, V, W$ and $p$, the boundary conditions are easily inserted. This means that at a wall, normal velocity component is zero and tangential components equal the velocity of the wall (usually zero). When velocity components are imposed on a boundary, the condition on pressure correction is the zero gradient type. For scalars $(\Phi s)$ the boundary condition is fixed value, fixed gradient or a mixed condition, all dependent on the physical requirement. 


\subsubsection{Non-linearities}

The set of algebraic equations to be solved is only nominally linear. This means that special measures must be taken to secure numerical stability of the solution. This is done by under-relaxation in a steady state problem and by imposing limits to the time step taken in a transient problem. Under-relaxation means that only part of the implications of the algebraic equation is accepted. This means

$$
\Phi_{a}=\alpha_{\Phi} \Phi_{a e}+\left(1-\alpha_{\Phi}\right) \Phi_{\text {old }}
$$

Here, $\alpha_{\Phi}$ is the under-relaxation factor for the variable $\Phi$ in question. Typical values for a sought steady solution are $\alpha_{U}=\alpha_{V}=\alpha_{\mathrm{w}}=0.3$ to $0.5, \alpha_{p^{\prime}}=0.5$ to 0.8 and for other scalars $\alpha_{\phi}=0.5$ to 0.7 . For a combusting flow with variable density, underrelaxation of the density of $\alpha_{\rho}=0.5$ may also be necessary.

For a transient situation, all $\alpha_{\phi}$ are set equal to 1.0 except $\alpha_{p^{\prime}}$ and $\alpha_{\rho}$ which are set to approximately 0.8 . The time steps are, on the other hand, limited by the Courant criteria, which says that the Courant number

$$
N_{c}=\frac{\Delta t \cdot c_{t}}{\Delta x}
$$

should be smaller than a given number usually in the order of unity. The typical velocity, $c_{t}$, to be used in the expression should be the absolute value of the local flow velocity for subsonic flows and the local sound speed for compressible supersonic flows.

\section{Computations and discussions}

The method described above is incorporated into a two-dimensional computer code presented by Hjertager (1982 a), and applied to study the following cases: The first is the shock tube problem that allows the model to be tested against known analytical solutions.

The second is calculation of flame and pressure development in the presence of turbulence inducing obstacles, a problem of great importance in accidental gas explosions.

\subsection{Shock tube}

The geometry and notation for this calculation are given in Fig. 2.

A $1 \mathrm{~m}$ long tube is initially divided into a high pressure and a low pressure region separated by a diaphragm. At time equals zero, the diaphragm is removed and two pressure waves are generated: A compression wave (shock) moving to the right and an expansion wave (rarefaction) moving to the left (Fig. 2). To calculate this problem a one-dimensional grid layout is chosen. The gas is treated as an ideal non-reactive gas with molecular weight equal to 29 and the ratio between specific heats equal to $1 \cdot 4$. Since this is a non-reacting situation, only equations for conservation of mass (2), momentum (3) and energy (4) need to be solved. The Courant number, defined as $c_{4} . \Delta t / \Delta x$, is nominally chosen to be $0 \cdot 2$.

Figure 3 shows the grid number dependence on the pressure distribution at time equals $0.591 \mathrm{~ms}$. It can be noted that the shock front position is well predicted even for coarse grids, whereas the steepness is more dependent of number of grid points. 


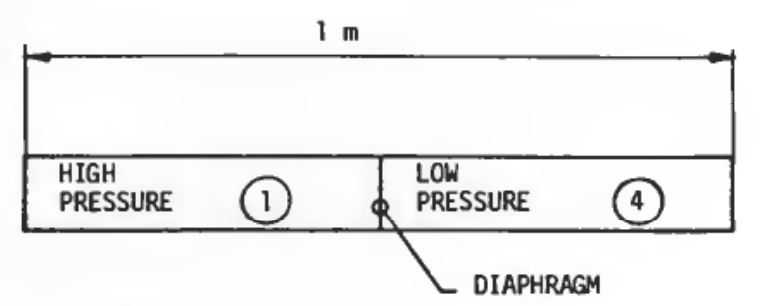

a. TIME $=0$

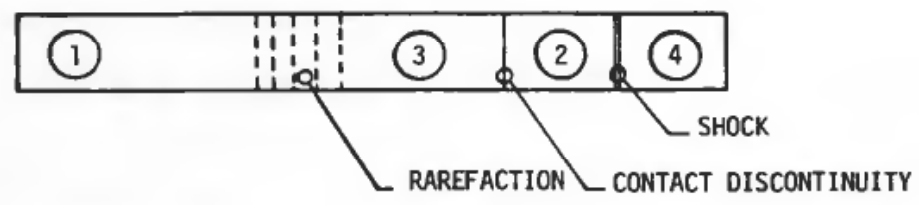

b. TIME $>0$

Figure 2. Geometry and notation for the shock tube problem.

This is the usual feature for most methods which treat shock fronts in fixed grid systems. The only simple remedy available for increased accuracy in such problems, is to increase number of points until acceptable grid independence is reached.

Figure 4 shows a comparison between exact solutions from Harlow and Amsden (1971) and predictions of shock pressure ratio and Mach number as a function of initial pressure ratio. For pressure ratios tested, from 1 to 20 , the agreement is satisfactory for both quantities. Calculations are also performed to test the stability of the numerical scheme. The Courant number is varied and converged solutions are obtained for values up to approximately $3 \cdot 0$. This is far beyond what any explicit method can perform.

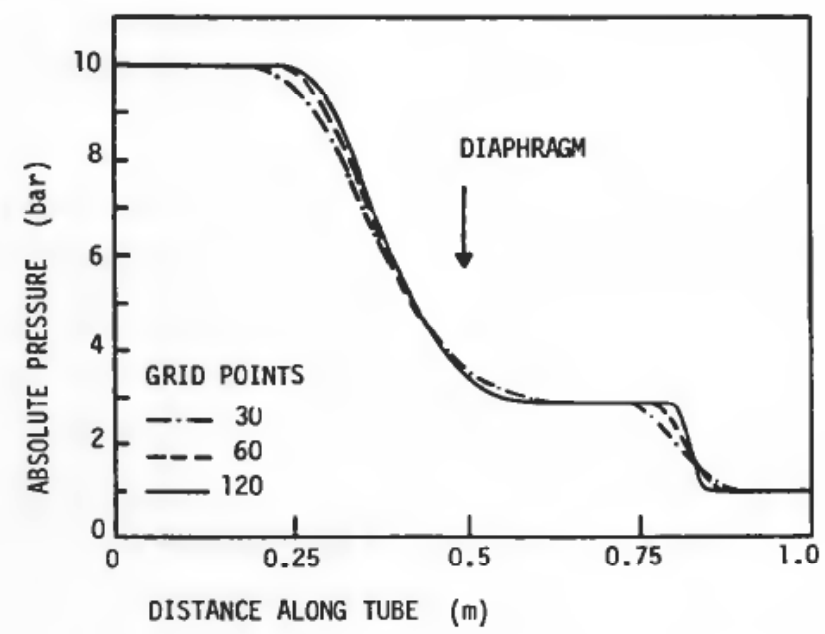

Figure 3. Grid dependence on pressure profile along the shock tube. Time equals $0.591 \mathrm{~ms}$. 


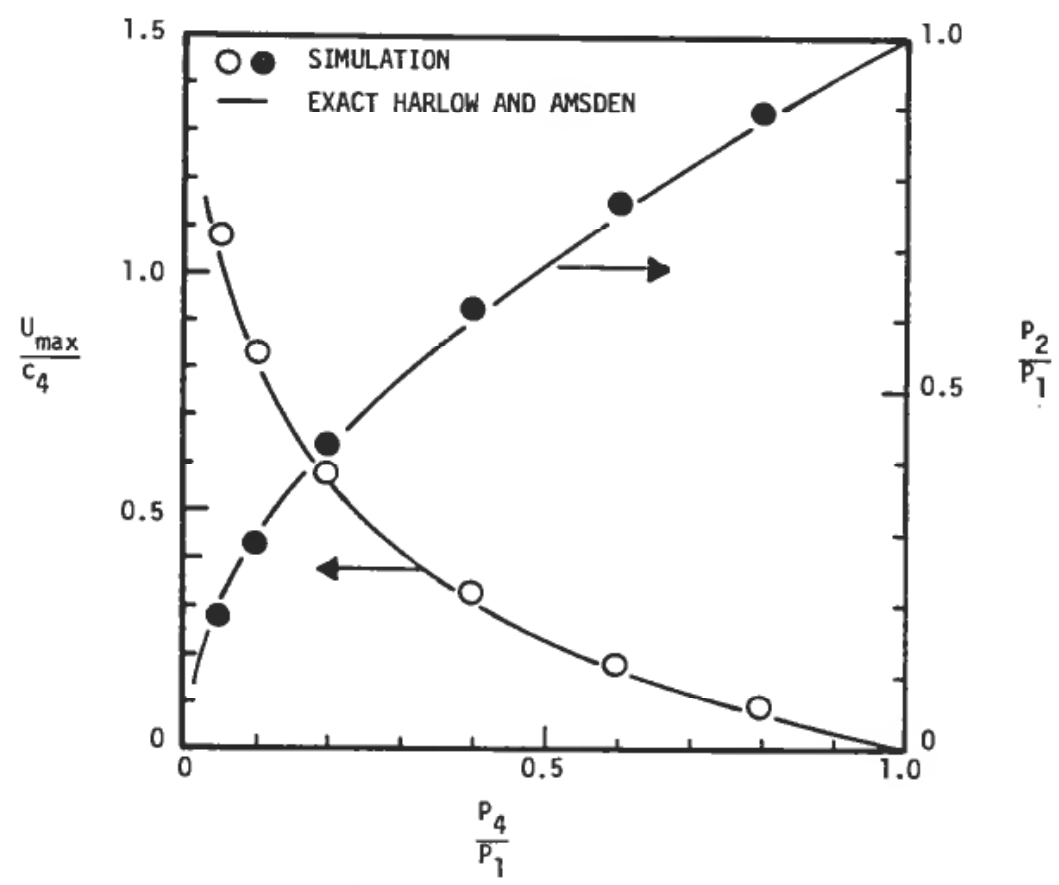

Figure 4. Variation of shock pressure ratio $\left(P_{2} / P_{1}\right)$ and Mach number $\left(U_{\max } / c_{4}\right)$ behind shock with initial pressure ratio $\left(P_{4} / P_{1}\right)$.

\subsection{Flame acceleration by turbulence}

To be able to predict consequences of gas explosions in integrated industrial systems, the problem of flame and pressure development in turbulent environments must be addressed. Several experiments have recently been performed to investigate such problems.

The qualitative mechanisms for this flow, turbulence and combustion interaction are fairly well established, but there is lack of quantitative methods to predict what happens in various geometries.

The present computation method is aimed at handling this complex interaction. The particular geometry that has been considered so far is a tube with axisymmetric repeated obstacles. This problem was studied by Moen et al. (1982) using methane/ air, and Hjertager et al. (1984) using propane/air in a large-scale tube facility situated at Sund, close to Bergen, Norway. The tube was $10 \mathrm{~m}$ long and had a diameter of $2.5 \mathrm{~m}$ and thus a volume of $50 \mathrm{~m}^{3}$. The ignition end was closed and the downstream end open. Turbulence-inducing rings of various widths and number were inserted inside the tube. In the present calculation we focus only on one geometrical configuration, namely 5 equally spaced rings, each blocking off 16,30 and $50 \%$ of the free tube area. The calculation domain is shown in Fig. 5 together with grid layout and dimensions. 30 grid points are used in the $x$-direction and 15 in the radial direction. The Courant number based on the unburnt condition was $0 \cdot 5$. The initial gas composition was a stoichiometric mixture of methane-air or propane-air and ignition was initiated by a planar source at the closed end (Fig. 5). The chemical induction time data has been taken from Burcat et al. (1972). No attempt was made to model details of the ignition process or the laminar to turbulent flow transition. 


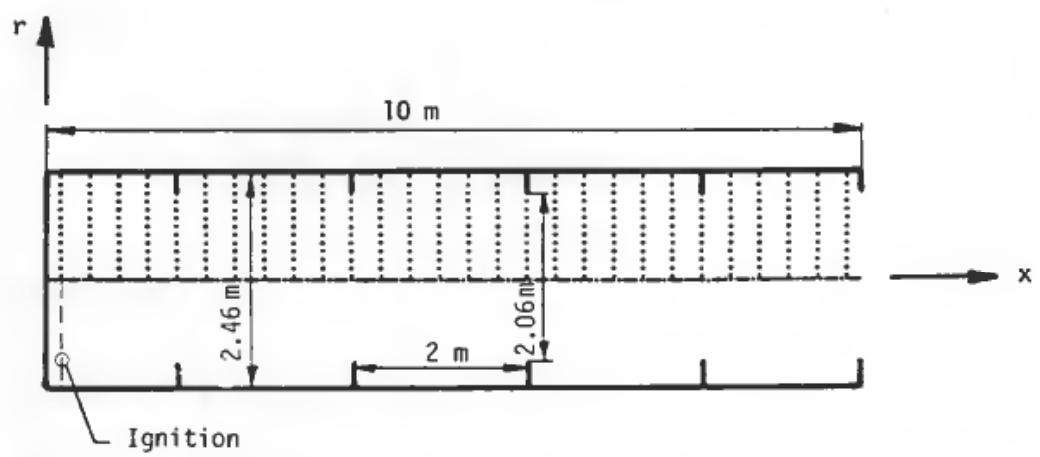

Figure 5. Geometry and grid layout for the flame acceleration problem.
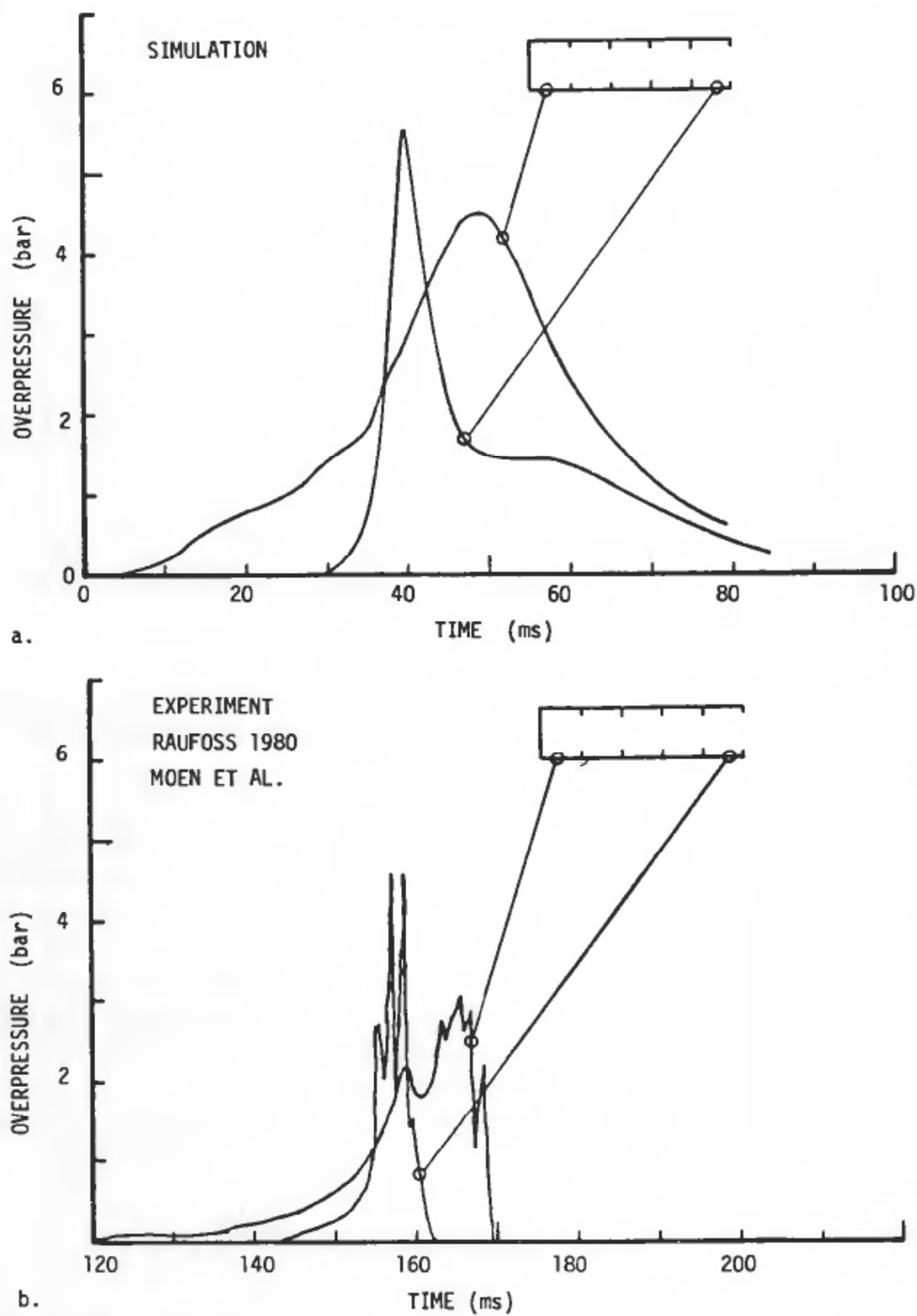

Figure 6. Variation of pressure with time for two positions along the tube. Prediction (a) and experiment $(b)$. 
In fact, the flow was treated as being in the turbulent regime from the start, and therefore, the turbulence and combustion models of paragraphs 3 and 4 were applicable. The ignition was accomplished by stating that $10 \%$ of fuel at the closed end of the tube was burnt and that the reaction rate and effective viscosity was dependent on the initial value of the dissipation rate $\varepsilon_{0}$. This value was varied until the resulting pressure and flame development at later times were almost independent of these initial conditions. The reason for this being valid, is the strong positive feedback mechanism which comes into play at the later stages of the process.

Figures $6(a)$ and $(b)$ show a comparison between predictions and experiments for two typical pressure traces versus time, one recorded near the ignition end, and one near the down-stream end of the tube. We can see that the general trends are reproduced in the calculations. This means increased pressure rise and peak pressure at the exit end of the tube as compared to the start end, and the later occurrence of the pressure peak at the start rather than at the exit. The largest discrepancies between experiment and simulation are found in the pressure decay period. A possible explanation to this may be the fact that Moen et al. (1982) used a plywood ring at the exit end, whereas the other rings were made of steel. This means that the last ring was destroyed in each experiment, possibly giving rise to a faster pressure
decay.

In Figs. $7(a)$ to $(j)$ some details of various stages in the flame and pressure propagation are depicted. For each point of time stated, distributions in space of the following four quantities are given. From top to bottom these are velocity vectors, pressure, concentration of combustion product and reaction rate. The meaning of the different vectors and contours is as follows: The largest velocity vectors displayed are $300 \mathrm{~m} / \mathrm{s}$ and if higher vectors occur, only this maximum is shown. For the flame contours the full line represents $50 \%$ of fully burnt condition, whereas the other two contours represent $75 \%$ burnt (broken line) and $25 \%$ burnt (broken and dotted line).

The other two quantities (pressure and reaction rate) are represented by contours scaled to the maximum value. This means that full lines represent $95 \%$, broken lines $75 \%$, broken dotted lines $50 \%$ and dotted lines $25 \%$ of the maximum quantity.

Figures $7(a)$ and $(b)$ show the initial phases after ignition. Pressure waves are sent ahead of the flame, which is planar at these two times., In Figs. $7(c)$ and $(d)$ distortion of the flame due to the influence of the first obstacle is observed. The peak reaction rate is located in the centre in Fig. $7(c)$, but in Fig. $7(d)$ the formation of large rates of reaction in the shear layer is seen. This again is confirmed by the pressure contours, which now have their maximum in the pocket (Fig. $7(d)$ ).

Occurrence of highest pressure and rates of combustion in the shear layers of the pockets are also evident in Figs. $7(e),(f)$ and $(g)$. The effect of turbulence enhancement on combustion and turbulent transport in the shear layer are depicted in Figs. $7(f)$ and $(g)$. In the first of these figures the leading flame is in the central part, whereas in Fig. $7(g)$ the flame is so distorted that the leading front is in the shear layer. Note that the peak rate of combustion in Fig. $7(g)$ is approximately 40 times larger than in the initial stages of propagation (Fig. 7 (a)). After the flame has reached the outlet, the pressure becomes more constant in the various cross sections, and from Fig. 7 (i) onwards the main process which occurs is venting and thus pressure relief. Although some combustion still occurs in the pockets (Fig. 7 (i) and (j)) the rate is much lower than during the most vigorous periods (Figs. $7(f)$ to $(g)$ ). 

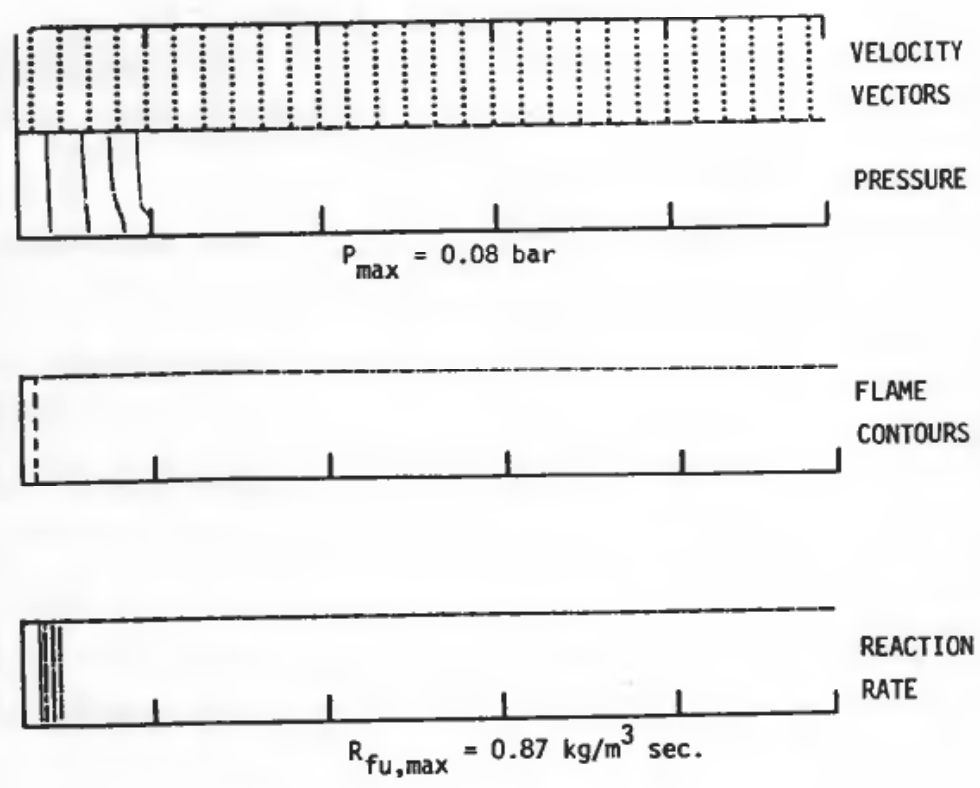

a) TIME $=6.0 \mathrm{~ms}$
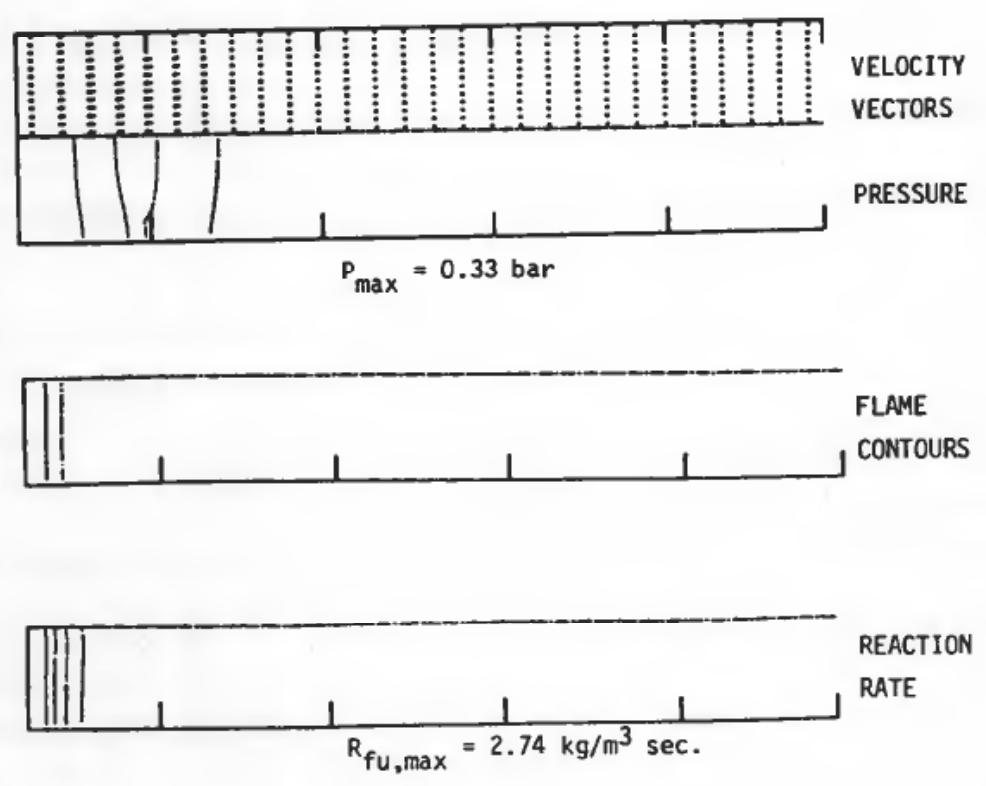

b) TIME $=11.9 \mathrm{~ms}$

Figure $7(a)-(b)$. Velocity vectors, pressure-, flame- and reaction rate contours for various time intervals. Predictions. 

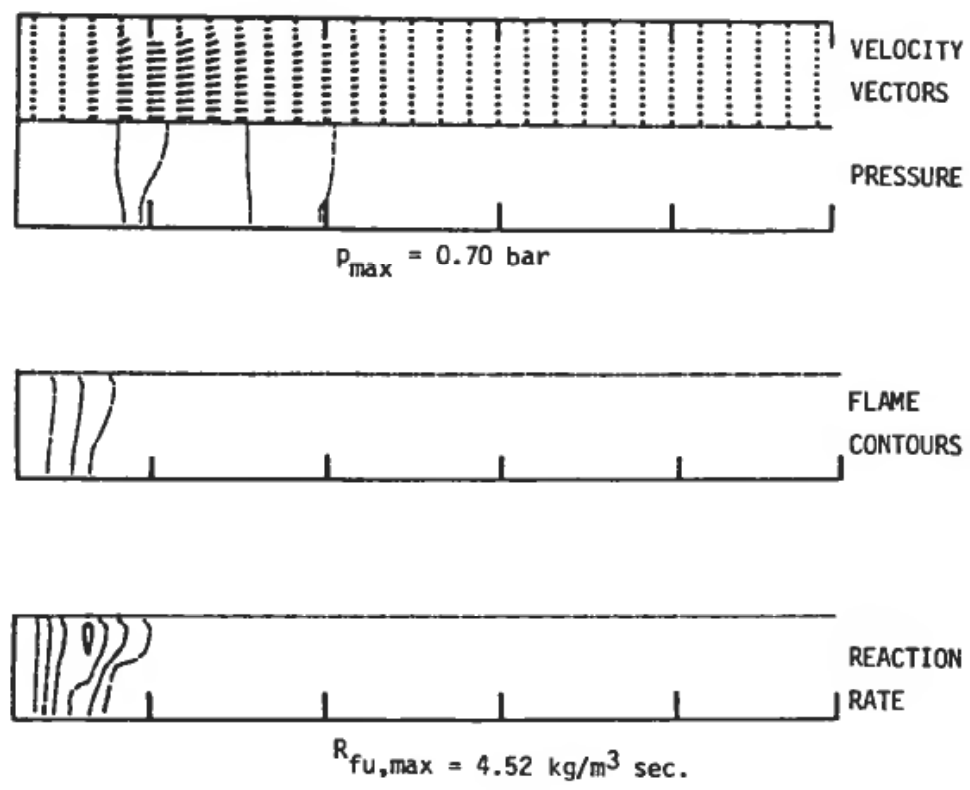

c) TIME $=17.9 \mathrm{~ms}$
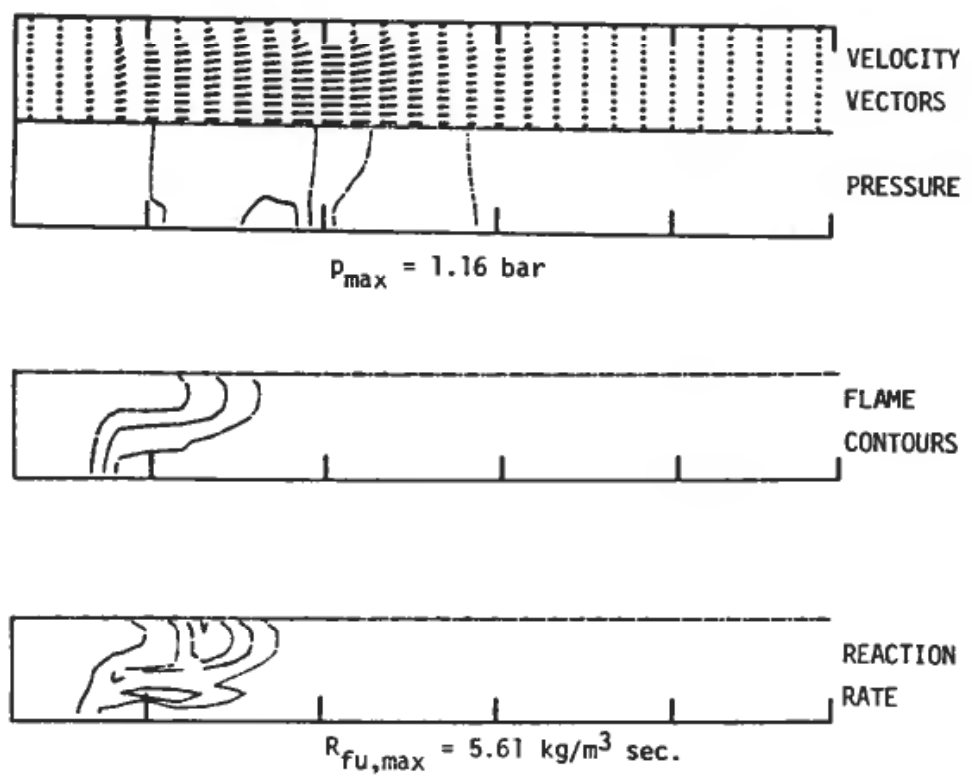

d) TIME $=23.8 \mathrm{~ms}$

Figure $7(c)-(d)$. Velocity vectors, pressure-, flame- and reaction rate contours for various time intervals. Predictions. 

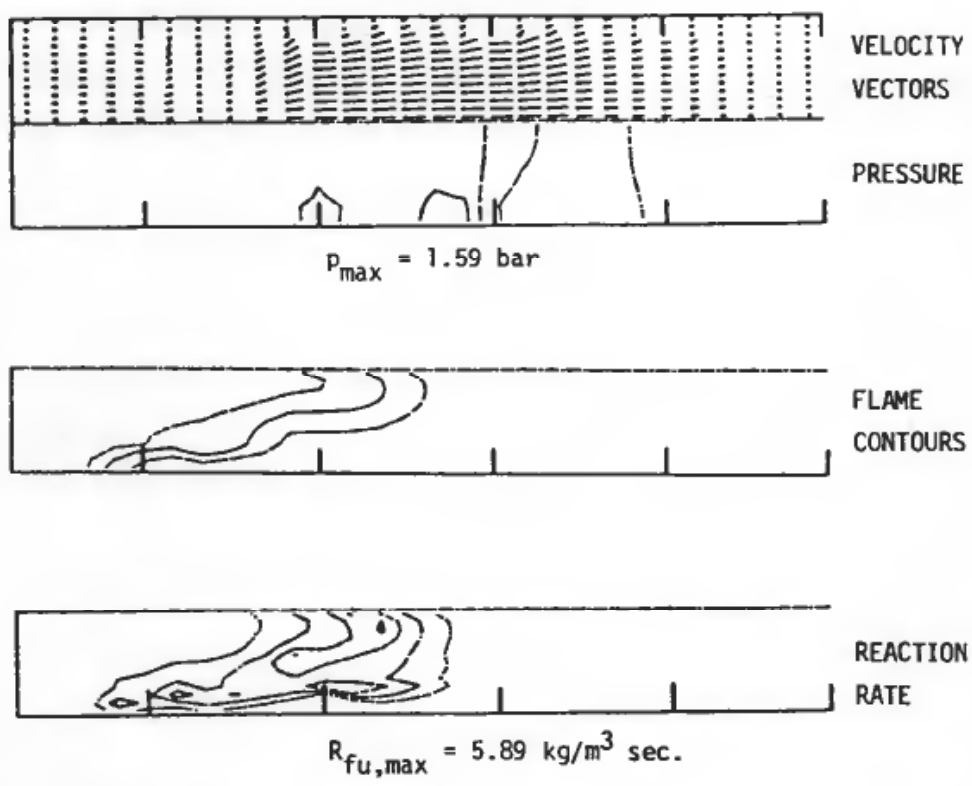

e) TIME $29.8 \mathrm{~ms}$
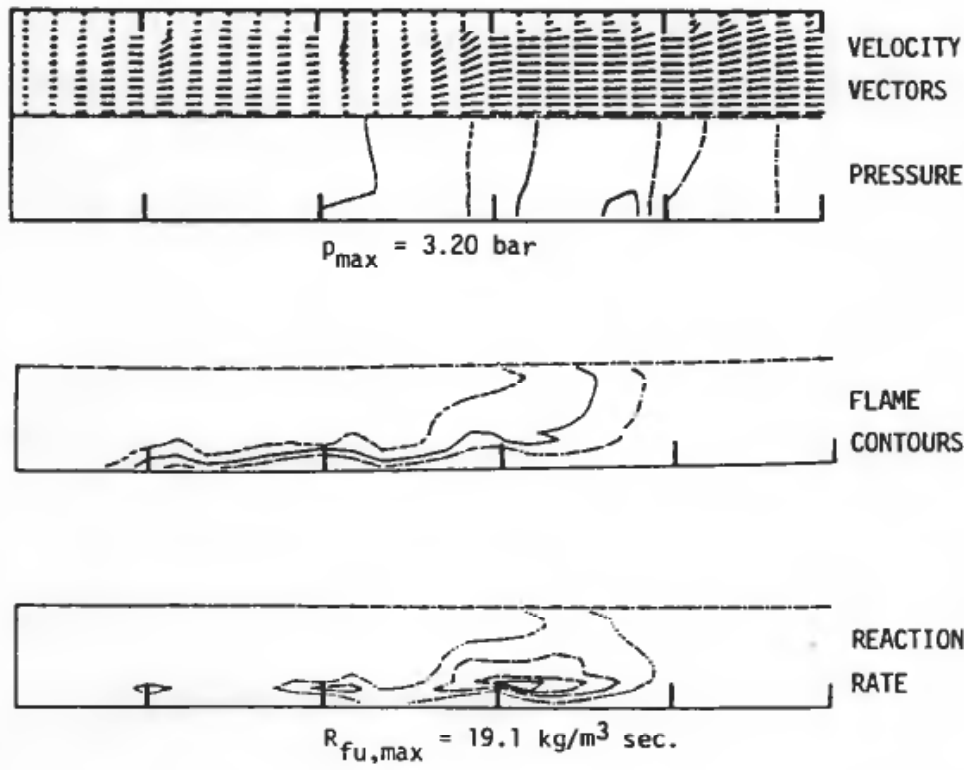

f) TIME $=35.7 \mathrm{~ms}$

Figure $7(e)-(f)$. Velocity vectors, pressure-, flame- and reaction rate contours for various time intervals. Predictions. 

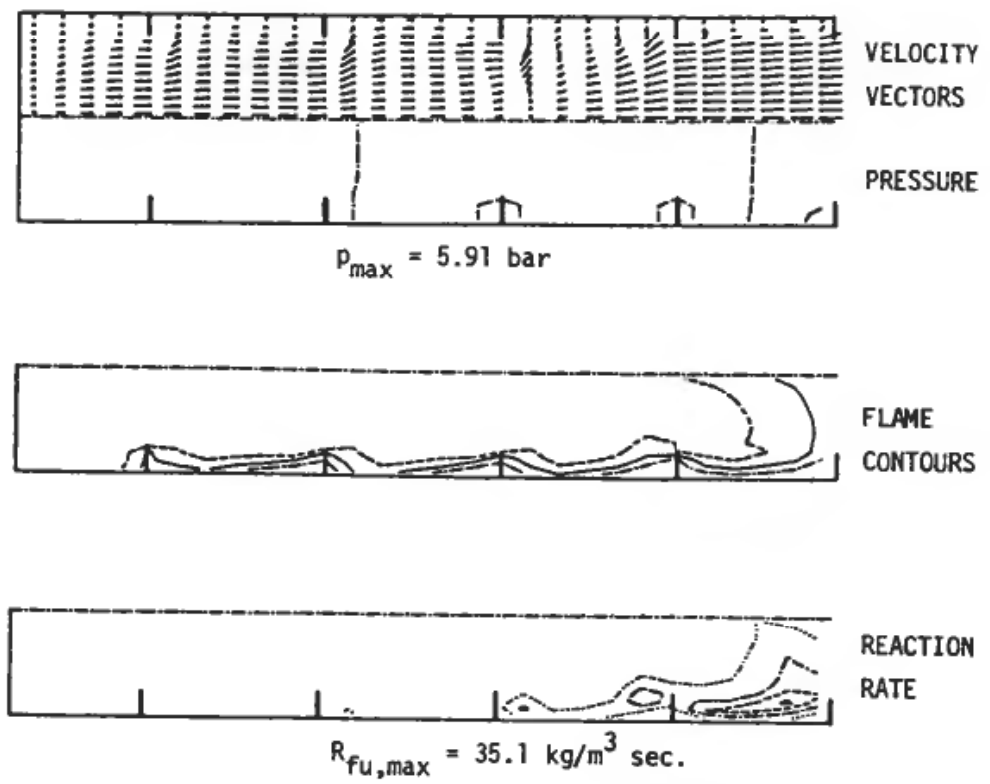

g) TIME $=39.9 \mathrm{~ms}$
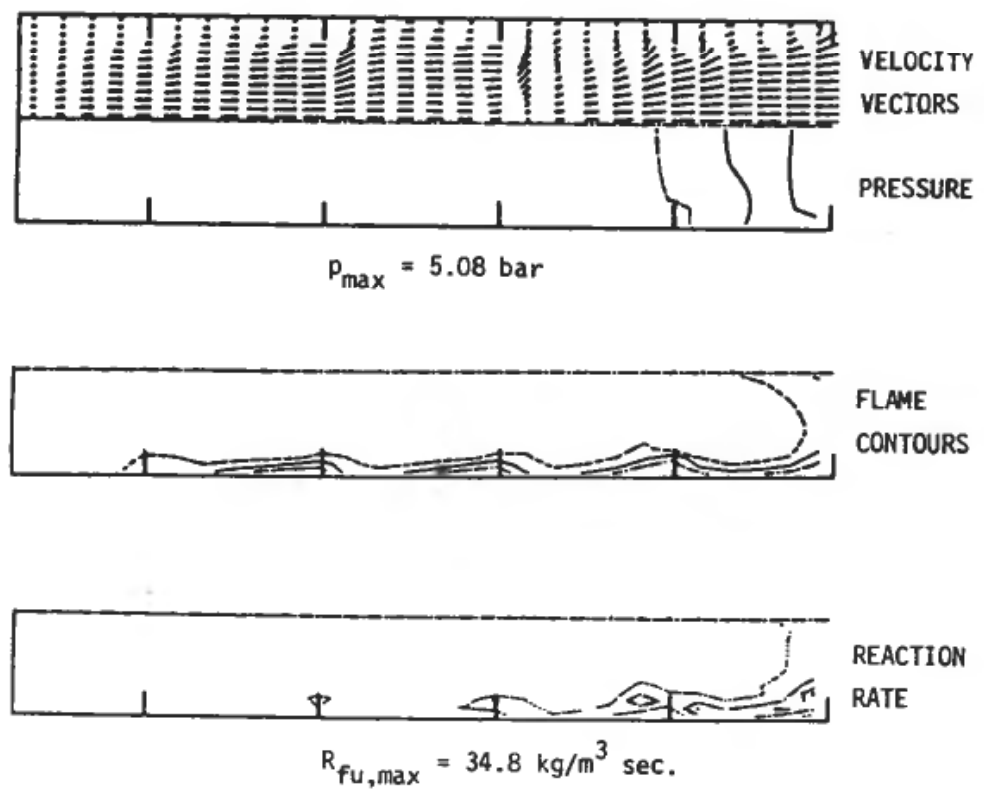

h) TIME $=41.1 \mathrm{~ms}$

Figure $7(g)-(h)$. Velocity vectors, pressure-, flame- and reaction rate contours for various time intervals. Predictions. 

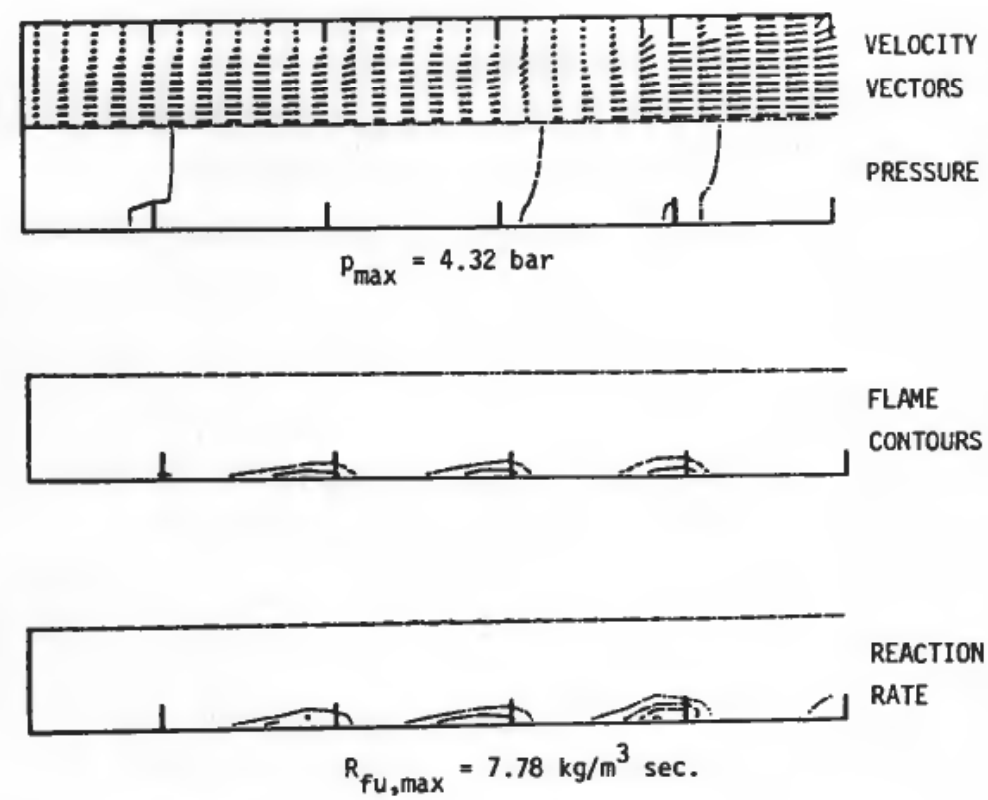

i) TIME $=46.9 \mathrm{~ms}$
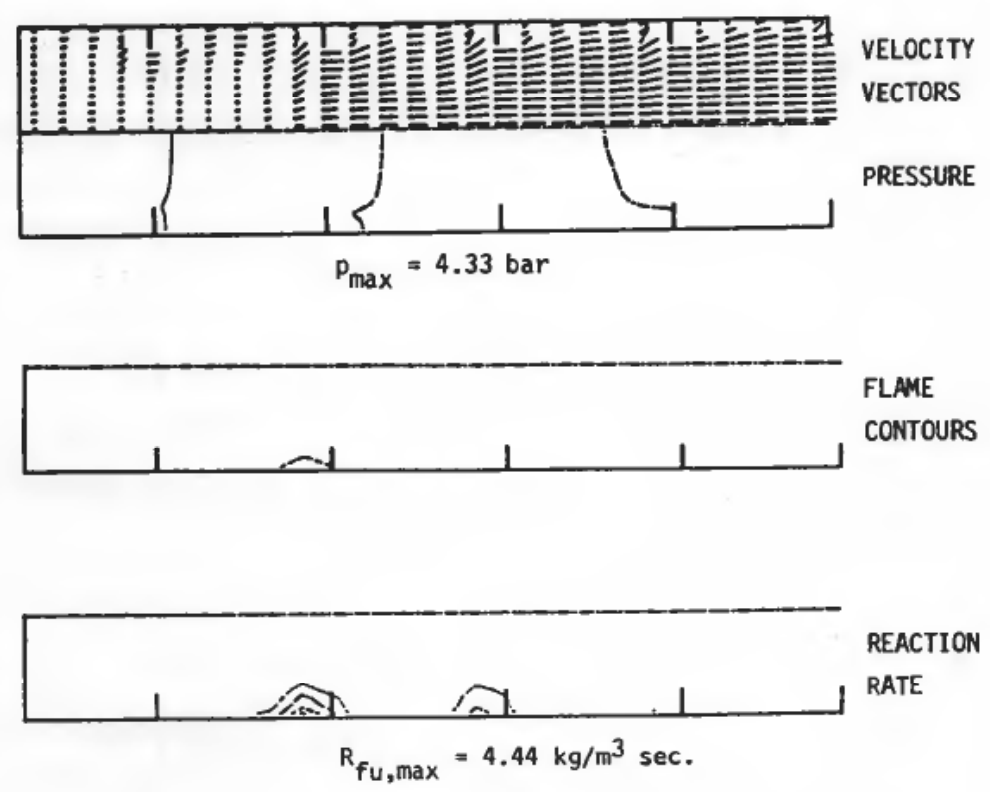

j) TIME $=52.2 \mathrm{~ms}$

Figure $7(i)-(j)$. Velocity vectors, pressure-, flame- and reaction rate contours for various time intervals. Predictions. 
Figure 8 shows comparisons between predicted and measured flame speeds along the centre line of the tube. The general features of the flame propagation are reproduced, that is a wavy increase of speed. These waves are due to the alternating acceleration and deceleration of the flow through the ring orifices.
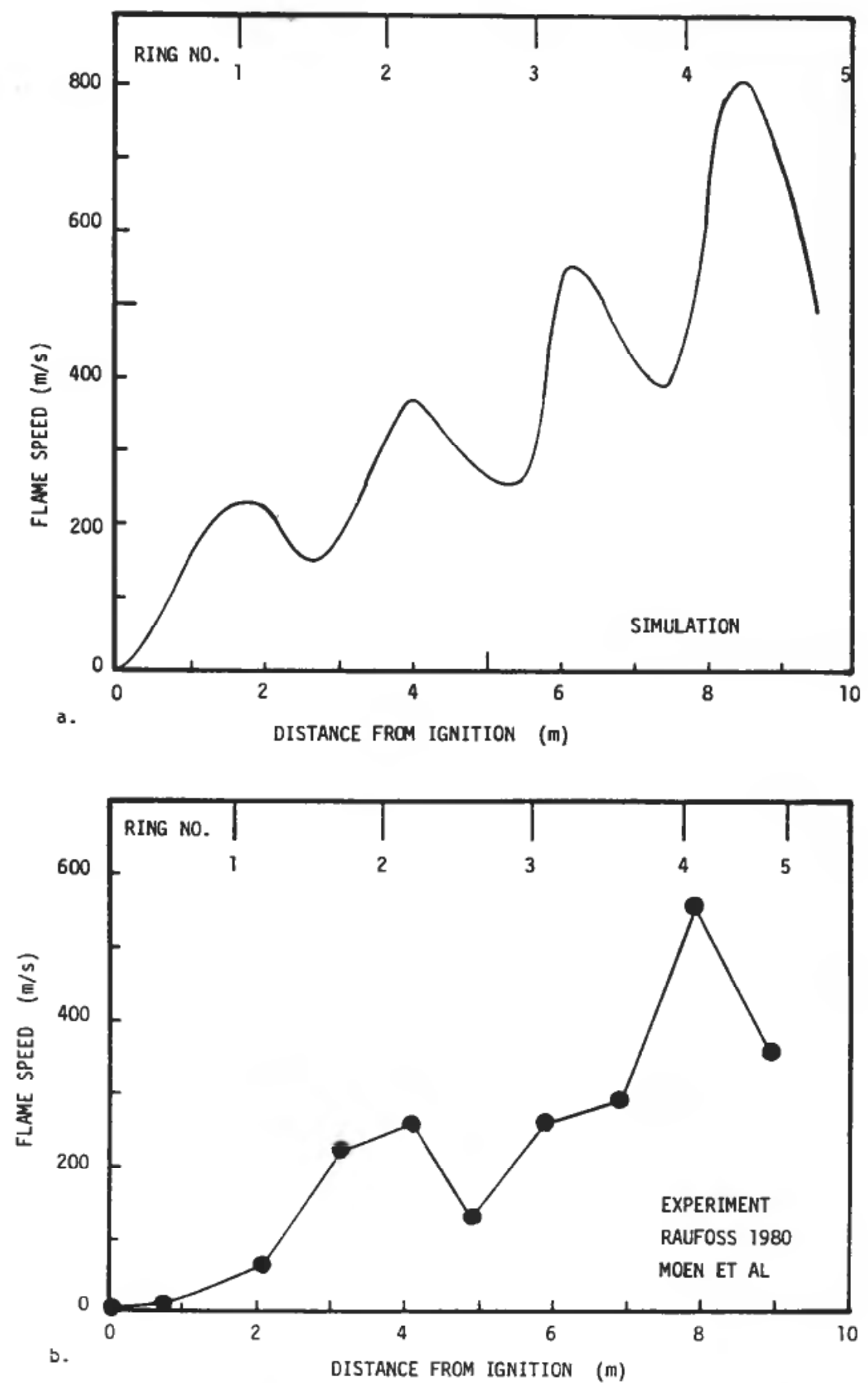

Figure 8. Variation of centre line flame speed with distance from ignition. Prediction (a), experiment $(b)$. 
Figure 9 shows a comparison between experiments and predictions of peak pressures versus blockage ratio $\left(B R=\left(1-(d / D)^{2}\right)\right.$ for methane-air and propane-air mixtures. The Fig. shows that the large difference in peak pressures between methane-air and propane-air explosions is fairly well predicted. The present prediction method also gives the correct behaviour of pressure versus blockage ratio. There is, however, some underprediction for propane-air at blockage ratio 0.5 . It should also be mentioned that the original combustion rate model (Magnussen and Hjertager, 1976) would only show a $20 \%$ difference between methane and propane. This clearly demonstrates that changes in only thermodynamic properties and use of the infinite chemical kinetics assumption are not capable of reproducing the experimental differences between methane-air and propane-air explosions.

A severe test for any prediction method is to see the predicted effect in various scales of the geometry. This has been done for the present geometry and the result is shown in Fig. 10. This Fig. shows the variations of the peak pressures inside the tube with linear scaling factor. A strong increase of pressures from laboratory-scale to large scale is observed, whereas further pressure increase with larger scales beyond approximately a scaling factor 1 for propane and a scaling factor of 10 for

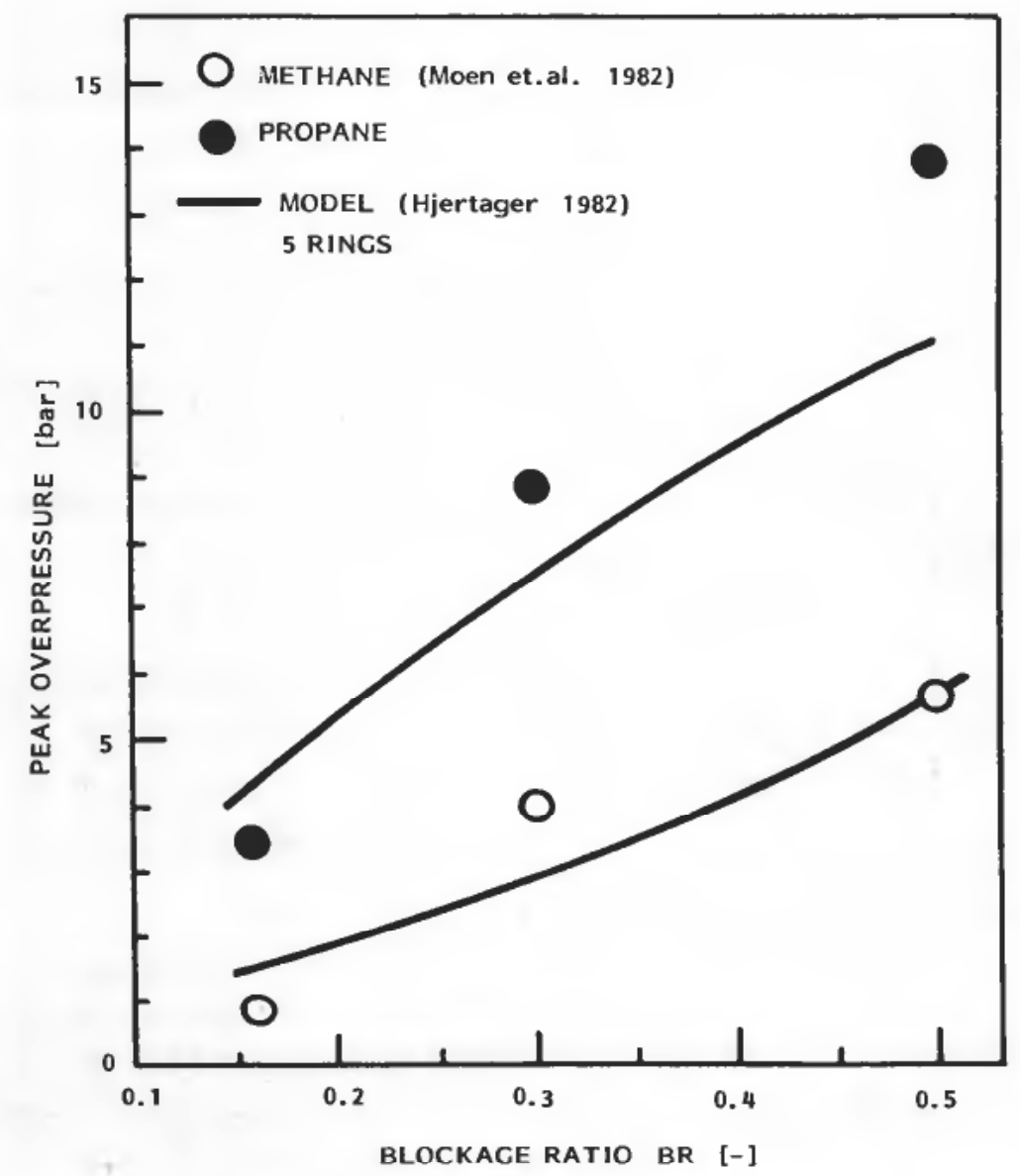

Figure 9. Peak measured (Moen et al., 1982; Hjertager et al., 1983) and predicted pressures in the $50 \mathrm{~m}^{3}$ combustion tube as function of blockage ratio, B.R. $=1-(d / D)^{2}$. Propane-air and methane-air mixtures. 


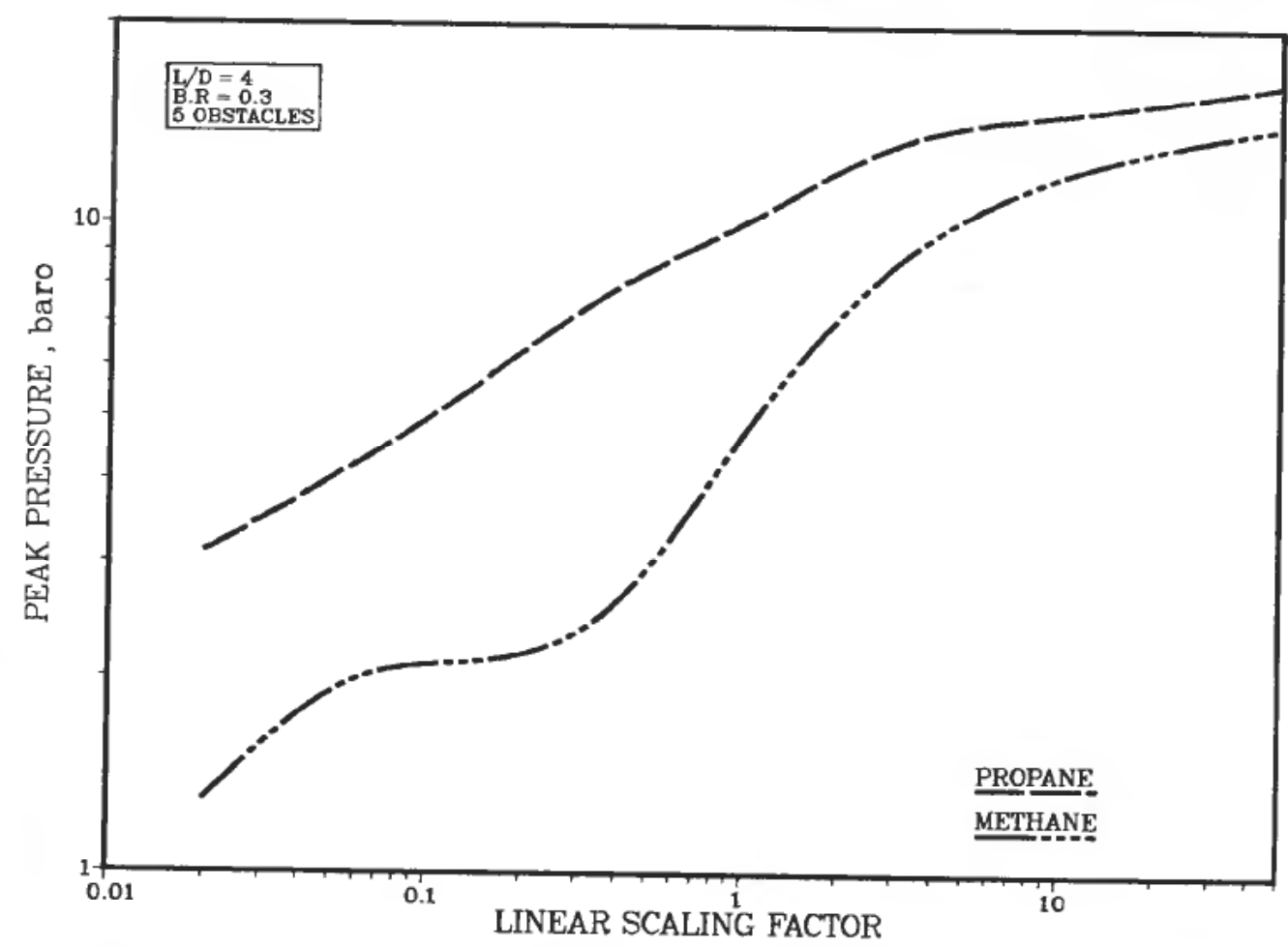

Figure 10. Variation of peak overpressure in stoichiometric mixtures of methane-air and propane-air with scaling. Scaling factors of 1.0 indicates $10 \mathrm{~m}$ of flame travel over 5 obstacles.

methane is moderate. Moen (I. O. Moen, private communications, 1980) conducted a small-scale experiment (Scale 1/16) of the $50 \mathrm{~m}^{3}$ Sund tube using methane/air and found that the peak overpressures were in the order of $0.5 \mathrm{bar}$, which is comparable to what the present method predicts.

\section{Conclusions}

A computer simulation method for dealing with compressible turbulent reacting flows has been presented. The calculation method has been tested for transient compressible flows. The results of testing against available analytical solutions for the shock tube problem are good. Furthermore, the problem of turbulent flame acceleration by obstacles has been modeled and comparison of predicted pressure pulses, flame speed and pressure load scaling with available experimental data is good. The predicted peak pressures are in general in good agreement with corresponding largescale and small-scale experimental data. This shows that rate of combustion in shear produced turbulence is mainly dependent on rate of micro mixing between turbulent eddies. It is also shown that the chemical kinetics of the system have to be considered in fast turbulent deflagrations to be able to correctly predict flame- and pressure development in different fuel-air mixtures. However, the computation method for compressible reacting flows must be tested in various other geometrical configurations to further investigate the potential of the model to predict explosion effects in real-life geometries. Turbulence measurements of various quantities in transient combusting flows are greatly needed in order to refine the various parts of the model. 


\section{ACKNOWLEDGMENTS}

The author gratefully acknowledges financial support from the oil companies BP Petroleum Development Ltd., Norway, Elf Aquitaine Norge A/S, Esso Exploration and Production Norway Inc., Mobil Exploration Norway Inc., Norsk Hydro and Statoil.

\section{REFERENCES}

Boni, A. A., Chapman, M. and SCHNeYer, G. P. (1976). Computer simulation of combustion processes in a stratified charge engine, Acta Astronautica. 3, 293-307.

Burcat, A., Crossley, R. W. and SCHELler, K. (1972). Shock tube investigation of ignition in ethane-oxygen-argon mixtures. Combustion and Flame, 18, 115-123.

BUTLER, T. D. and O'ROURKE, P. J. (1976). A numerical method for two-dimensional reacting flows, 16th International Symposium on Combustion (Combustion Institute: Pittsburg) pp. 1503-1515.

Harlow, F. H. and Amsden, A. A. (1971). Fluid Dynamics, A Los Alamos Scientific Laboratory monograph, LA-4700.

HJeRTAGER, B. H. (1982a). Simulation of transient compressible turbulent reactive flows, Combustion Science and Technology, 27, 159-170. (1982b). Numerical simulation of turbulent flame and pressure development in gas explosions, Fuel-air Explosions, SM Study No. 16 (University of Waterloo Press, Ontario, Canada) pp. 407-426.

HJertaGer, B. H., Fuhre, K., PARKER, S. J. and BAKKE, J. R. (1984). Flame acceleration of propane-air in a large-scale obstructed tube, 9 th International Colloquium on Dynamics of Explosions and Reactive Systems, Poitiers, France, 3-8 July, 1983. Prog. AIAA, 94, 504-522.

HJeRTAGER, B. H. and MAGNussen, B. F. (1976). Computation of some three-dimensional laminar incompressible internal flows, Proceedings 1976, Heat Transfer and Fluid Mechanics Institute (Stanford University Press) pp. 436-451.

Khalil, E. E., Spalding, D. B. and Whitelaw, J. H. (1975). The calculation of local flow properties in two-dimensional furnaces, Int. J. Heat Mass Transfer, 18, 775-791.

LAUNDER, B. E. and SPALDING, D. B. (1974). The numerical computation of turbulent flows, Computer Methods in Applied Mechanics and Engineering, No. 3, 269-289.

MAGNUSSEN, B. F. (1981). On the structure of turbulence and a generalized eddy dissipation concept for chemical reaction in turbulent flow, Proc. 19th AIAA Aerospace Science Meeting, St. Louis, Missouri, January 12-15, 1981.

MAGNuSSEN, B. F. and HJERTAGER, B. H. (1976). On mathematical modelling of turbulent combustion with special emphasis on soot formation and combustion, 16th International Symposium on Combustion, (Combustion Institute, Pittsburg), pp. 719-729.

Moen, I. O., LeE, J. H. S., HJerTAGer, B. H., FuHRe, K. and ECKHOFF, R. K. (1982). Pressure development due to turbulent flame propagation in large-scale methane-air explosions, Combustion and Flame, 47, 31-52.

Oran, E., YounG, T. and BorIS, J. (1978). Application of time-dependent numerical methods to the description of reactive shocks, 17th International Symposium on Combustion, (Combustion Institute: Pittsburg), pp. 43-53.

PATANKAR, S. V. (1981). A calculation procedure for two-dimensional elliptic problems, Numerical Heat Transfer, 4, 409-426.

Patankar, S. V. and Spalding, D. B. (1972). A calculation procedure for heat, mass and moment transfer in three-dimensional parabolic flows, Int. Journal of Heat and Mass Transfer, 15, 1787-1806.

Radhkrishnan, K., HeYwood, J. B. and Tabaczynski, R. J. (1981). Premixed turbulent flame blowoff velocity correlation based on coherent structures in turbulent flows, Combustion and Flame, 12, 19-23.

SPALDING, D. B. (1971). Mixing and chemical reaction in steady confined turbulent flames, 13th International Symposium on Combustion (Combustion Institute: Pittsburg), pp. 649-657. 